\title{
Fonctions pédagogiques et implications énonciatives de ressources professorales multimodales
}

Le cas de la bimanualité et de l'ubiquité coénonciative

\section{Brahim Azaoui}

\section{(2) OpenEdition}

Édition électronique

URL : http://journals.openedition.org/rdlc/729

DOI : $10.4000 /$ rdlc. 729

ISSN : 1958-5772

Éditeur

ACEDLE

Référence électronique

Brahim Azaoui, «Fonctions pédagogiques et implications énonciatives de ressources professorales multimodales », Recherches en didactique des langues et des cultures [En ligne], 12-2 | 2015, mis en ligne le 13 septembre 2016, consulté le 10 décembre 2020. URL : http://journals.openedition.org/rdlc/729 ; DOI : https://doi.org/10.4000/rdlc.729

Ce document a été généré automatiquement le 10 décembre 2020.

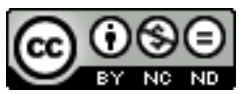

Recherches en didactique des langues et des cultures is licensed under a Creative Commons AttributionNonCommercial-NoDerivatives 4.0 International License 


\section{Fonctions pédagogiques et implications énonciatives de ressources professorales multimodales}

Le cas de la bimanualité et de l'ubiquité coénonciative

Brahim Azaoui

\section{Introduction}

1 Les évènements de la classe sont fondamentalement marqués par une sorte de plurivocalité constituée notamment de polylogues et d'échanges polyfocalisés que l'enseignant est parfois amené à réguler simultanément pour servir son projet pédagogique ou organiser les interactions didactiques. Les ressources verbales dont l'enseignant dispose dans son répertoire didactique (Cicurel, 2002, 2011 ; Bigot, Blondel, Cadet \& Causa, 2004; Cadet, 2006; Cadet \& Causa, 2005; Causa, 2012) ne peuvent objectivement pas permettre à elles seules de répondre à ces exigences. Aussi, il convient de s'intéresser également aux stratégies multimodales auxquelles fait appel l'enseignant. Or, si la définition de l'agir professoral (Cicurel, 2011: 119) renvoie explicitement au caractère multimodal des actions enseignantes, force est de constater que ces ressources en classe sont encore peu renseignées dans la littérature scientifique.

2 C'est dans cette perspective que se place la présente contribution. L'analyse que nous proposons se situe au croisement des travaux sur l'agir professoral en contexte classe et des études de la gestuelle (les "gesture studies"). En observant et en analysant sous un angle multimodal l'action professorale, nous avons pu mettre au jour diverses ressources auxquelles les enseignantes de nos corpus avaient recours pour gérer la plurivocalité des interactions didactiques et leur projet pédagogique.

3 Nous reviendrons dans un premier temps sur deux aspects de la réalité des interactions didactiques: la dimension plurivocale des échanges et la multimodalité des actions 
professorales. Dans un deuxième temps, nous présenterons les corpus sur lesquels s'appuie cette contribution, ainsi que notre démarche de transcription et d'annotation des corpus à l'aide du logiciel Elan (Sloetjes \& Wittenburg, 2008). Pour finir, nous exposerons les résultats de nos analyses, qui s'articulent autour de deux notions: la bimanualité et l'ubiquité coénonciative. Cette dernière sera abordée en considérant les enjeux énonciatifs en classe de français (langue première et seconde).

\section{Interactions didactiques et multimodalité des actions professorales}

Les interactions didactiques sont reconnues pour être un type d'interaction située bien défini (Cicurel, 2005: 182) qui repose sur une forme de plurivocalité. Qu'elle relève de l'histoire interactionnelle, de la dimension polylogale ou de la polyfocalisation, cette plurivocalité exige de l'enseignant une aptitude à gérer ces voix multiples s'il veut mener à bien son projet d'enseignement. Les études sur le geste pédagogique, ou plus largement sur la multimodalité de l'action professorale, laissent supposer que cette aptitude repose en partie sur les ressources multimodales dont l'enseignant dispose et qui constituent son répertoire didactique.

\section{Les interactions didactiques : une réalité plurivocale}

5 Il existe une certaine plurivocalité dans les interactions didactiques que l'on peut observer à plusieurs niveaux. D'une part, elles sont ancrées dans une historicité (Doyle, cité par Clanet, 2002 : 90) qui favorise les renvois discursifs d'un cours à l'autre. En effet, chaque séance s'inscrit dans une "histoire interactionnelle" (Vion, 1992: 99) qu'il convient de considérer pour comprendre les différents enjeux interactionnels et pédagogiques. Les voix du passé sont donc ponctuellement convoquées pour rappeler la chronologie du projet pédagogique en cours ou pour étayer certains propos. D'autre part, les interactions didactiques en classe de collège français mettent habituellement face-à-face une trentaine d'élèves et un enseignant. Aussi, dans le prolongement de la rupture théorique posée par la notion de trilogue, présentée par Kerbrat-Orecchioni \& Plantin (1995), Bouchard (1998) propose de considérer les interactions didactiques comme des "polylogues" pour rendre compte des échanges mettant en présence plus de trois participants.

6 Selon la typologie retenue par Grosjean \& Traverso (1998: 57), les polylogues didactiques entrent dans la catégorie des situations avec focalisation collective dans un cadre formalisé, dans lesquelles un animateur des échanges tient le rôle de coordinateur. Nous devons à Goffman (cité par Rivière \& Bouchard, 2011) la notion de focalisation, qu'il a distinguée en trois types : regroupements non focalisés, focalisés et polyfocalisés. Dans les premiers, nous retrouvons les regroupements de personnes qui ne partagent rien en commun, si ce n'est le lieu de la rencontre (une salle d'attente). Les regroupements focalisés réunissent des personnes qui partagent "un certain degré d'engagement mutuel" (Rivière \& Bouchard, $2011:$ n.p. ${ }^{1}$ ). Enfin, pour ces auteurs, les interactions didactiques sont un exemple de situations polyfocalisées qui "regroupent plusieurs foyers, mobiles, d'attention et d'interaction" (ibid.). La gestion de ces échanges polyfocalisés et polylogaux s'effectuent en faisant appel à une batterie de ressources multimodales sur lesquelles il convient à présent de revenir. 


\section{Ressources multimodales de l'action professorale}

Des travaux précurseurs traitant du non-verbal dans la communication quotidienne, comme ceux menés par Scheflen ou de Spiegel \& Machotka sur la posture (cités par Corraze, 1980 : 129-140) ou par Condon (1976) sur la "synchronie interactionnelle", ont permis de mieux comprendre la composante non verbale du langage humain dans les interactions. Les mimiques faciales plus spécifiquement participent de cette interaction, ne serait-ce que parce que c'est à ce niveau que sont produits la plupart des feedbacks (Ekman \& Friesen, 1969 : 94). Un élément des mimiques faciales a particulièrement retenu l'attention de nombreux chercheurs depuis une cinquantaine d'années: le regard. Ces travaux ont permis de mettre au jour sa fonction dans l'interaction (Kendon, 1967; Heylen, 2005), et notamment dans la désignation du destinataire du message (Cook, 1977 ; Corraze, 1980 ; Goodwin, 1981, 1996 ; Streeck, 1993).

Dans le domaine de la didactique, Foerster (1990) a analysé le rôle du regard en classe de langue. Elle a observé la fonction métacommunicative (évaluation de l'énoncé) et éventuellement communicative du regard entre apprenants et enseignants. Elle a par ailleurs souligné son importance dans la gestion des tours de parole. Kååntå (2007), s'intéressant spécifiquement aux questions en classe de langue et en cours de disciplines non linguistiques ${ }^{2}$, montre le rôle combiné du verbal, des gestes et du regard dans les stratégies enseignantes pour favoriser la contribution des élèves au cours.

Plus globalement, la question du geste intéresse tout particulièrement les études menées en didactique depuis une trentaine d'années. En France, Tellier s'y consacre en étudiant ce qu'elle nomme le "geste pédagogique" $(2006,2008 \mathrm{~b})$. Elle le définit comme "un geste des bras et des mains (mais il peut aussi être composé de mimiques faciales) utilisé par l'enseignant de langue dans un but pédagogique" (Tellier, 2008b: 41). Il nous semble que cette définition pourrait cependant être précisée. "Les gestes, effectués pour atteindre un but, [étant] organisés en fonction de ce but" (Calbris \& Porcher, 1989 : 34), ce qui fait du geste de l'enseignant un geste pédagogique n'est pas le contexte didactique dans lequel il est produit, et encore moins qu'il soit réalisé par un professeur comme l'indique Tellier ("par l'enseignant"). C'est essentiellement l'intention recherchée. Dès lors, nous pourrions envisager de situer les gestes pédagogiques sur un continuum selon le degré de didacticité du geste. Pour développer notre réflexion, nous ferons appel à la notion de "didacticité" présentée par Moirand (1993: n.p.). Cette auteure précise que pour caractériser la didacticité d'un discours il convient de s'intéresser au contexte d'énonciation, à la fonction du discours et à sa forme. L'association de ces trois conditions permet de "caractériser un discours didactique". Cela correspond au geste pédagogique tel que l'envisage Tellier. Or, Moirand (1993) poursuit son analyse en distinguant une "didacticité première", qui renvoie à des discours produits dans une institution scolaire et une "didacticité seconde" qui fait référence aux "discours dont l'objet premier n'est pas didactique, mais qui relèvent, pour une part (...) d'une intention de didacticité, que celle-ci soit réelle ou simulée ou feinte ${ }^{3 "}$. Il nous semble possible d'appliquer cette distinction à la gestuelle pédagogique. Ainsi, la gestuelle adaptée à l'étranger, telle qu'analysée par Adams (1998) en contexte non didactique, entrerait dans la catégorie "didacticité seconde". Dans une interaction interlingue, posée comme asymétrique au moins sur le plan linguistique, l'énonciateur natif adaptera - donc conscientisera davantage - sa gestuelle à son interlocuteur allophone pour lui faciliter la compréhension d'un terme, d'un propos, sans qu'il n'existe pour autant officiellement de 
relation enseignant/enseigné. Selon leur niveau de didacticité, nous pourrions donc situer les gestes sur un continuum en considérant différents éléments, tels que le contexte d'énonciation (contexte pédagogique ou non), l'intention didactique, les caractéristiques des gestes produits (conscientisation du geste, amplitude, durée, iconicité, etc.), voire le profil de l'individu (a-t-il suivi une formation sur la gestuelle ? estil un professionnel de l'enseignement?...).

Pour ce qui est du geste pédagogique en classe, Tellier (2006) a mis au jour trois fonctions qui font écho aux travaux de Dabène (1984) : animer, informer et évaluer. De nombreuses études se sont intéressées aux fonctions d'information et d'évaluation et il en est ressorti que la gestuelle est essentielle dans l'input fourni aux apprenants. Ces études ont en effet souligné l'impact de la gestuelle dans la mémorisation (Allen, 1995; Tellier, 2008a) ou dans la compréhension (Kellerman, 1992; Lazaraton, 2004; Sime, 2008; Tellier, 2009, 2010). Au niveau de la correction, des études ont montré le rôle que la gestuelle pouvait jouer pour favoriser le travail autocorrectif des apprenants (Schachter, 1981 ; TaleghaniNikzam, 2008).

11 Considérant, pour l'essentiel, les fonctions d'animation et d'évaluation dans la construction de normes scolaires, nous avons contribué à la compréhension des fonctions du geste pédagogique (Azaoui, 2014a). Ce travail s'adosse à un corpus de films de classe et de séances vidéoscopiques dont la présentation est l'objet de la partie suivante.

\section{Présentation des corpus}

\section{Un double corpus vidéo : interactions didactiques et vidéoscopie}

Nos corpus, recueillis selon une approche ethnographique de classe, rassemblent des interactions didactiques de deux enseignantes chevronnées de collège (EnsT et EnsM). Chacune enseigne sur des temps distincts le français langue seconde auprès d'Eana (Élèves Allophones Nouvellement Arrivés) et le français langue première face à des élèves francophones.

Ce corpus de classe a été complété par un ensemble de trois types de corpus vidéoscopiques filmés; dans un souci de clarté, nous présentons brièvement les trois mais seuls les deux premiers feront l'objet d'une analyse dans cette contribution. Durant le premier, l'autoscopie, l'enseignante (EnsM) s'observe et commente des extraits de ses cours de FL1 (Français Langue Première) et FLS (Français Langue Seconde). Le deuxième, l'hétéroscopie, permet aux élèves de cette enseignante d'observer et de commenter ces mêmes extraits de cours selon la classe à laquelle ils appartiennent (FLS ou FL1). Enfin, le troisième est l'autohétéroscopie (Joly, 2011 ; Azaoui, 2014b) : l'enseignante observe et commente les remarques émises par ses élèves lors des séances d'hétéroscopie. Si ces corpus possèdent une certaine pertinence dans une perspective de formation professionnelle (Azaoui, 2014b), ils ont également un intérêt non négligeable dans la compréhension des pratiques de transmission, qu'elles soient commentées par l'enseignante filmée ou par certains de ses élèves allophones et francophones. Nous nous évoquerons ici un exemple de corpus autoscopique. 


\section{Transcription et annotation multimodale}

14 Sur l'ensemble des interactions didactiques enregistrées, près de $6 \mathrm{~h} 30$ ont été intégralement transcrites et annotées à l'aide du logiciel multilinéaire d'annotation multimodale ${ }^{5}$ (Sloetjes \& Wittenburg, 2008). Notre typologie, présentée en détail ailleurs (Azaoui, 2014c), distinguait les gestes selon une approche mcneillienne (McNeill, 1992) :

Tableau 1 - typologie des gestes selon McNeill (1992)

\begin{tabular}{|l|l|}
\hline Battement & gestes rythmant la parole \\
\hline Déictiques & gestes de pointage \\
\hline Iconiques & gestes représentant un objet concret ou une action \\
\hline Métaphoriques & gestes représentant un concept, une idée \\
\hline Emblèmes & gestes fortement conventionnels et marqués culturellement \\
\hline
\end{tabular}

Nous avons adjoint à ces gestes les emblèmes pédagogiques, que nous avons définis comme les emblèmes propres/communs en situation de classe.

Pour ce qui est des mimiques, nous avons considéré diverses expressions faciales (Heylen et al., 2007), telles que les froncements de sourcils, les sourires ou les hochements de tête. En cours d'analyse, il nous a paru nécessaire d'intégrer à notre typologie le regard.

17 L'analyse qualitative de ces discours multimodaux en interaction a fait ressortir une compétence multimodale à gérer simultanément la dimension polyfocale des interactions, ce qui n'est pas sans soulever des questions en termes d'implication énonciative.

\section{Polyfocalisation, action multimodale et enjeux énonciatifs}

18 S'il convient de reconnaitre la simultanéité des échanges comme un élément définitoire des interactions didactiques, il s'agit également de s'interroger sur les actions que les enseignants mettent en œuvre pour gérer cette plurivocalité. Dans le cadre de notre travail plus global (Azaoui, 2014a), il était question d'observer comment les enseignantes parvenaient à manifester de l'intérêt pour les interventions tout en ménageant les normes linguistiques et interactionnelles.

19 Il ressort de cette analyse deux compétences complémentaires, la bimanualité et l'ubiquité coénonciative, qui renvoient pour chacune des enseignantes à leur style professoral, tel que le présentent Cicurel (2011: 166) et Azaoui (2014a: 64), à savoir la récurrence de pratiques pédagogiques ayant la même visée indépendamment du contexte d'enseignement. Sur le plan énonciatif, ces actions multimodales soulèvent la question du destinataire privilégié des réactions de l'énonciateur. 


\section{La bimanualité : lorsque l'enseignant se fait chef d'orchestre}

La bimanualité n'est pas à entendre ici uniquement comme la production gestuelle impliquant les deux mains, comme nous avons pu l'étudier ailleurs (Tellier, Azaoui \& Saubesty, 2012). Dans le cadre de ce travail, elle renverra à l'utilisation des deux mains pour produire deux gestes simultanément ou de manière légèrement différée. Chaque geste faisant partie de phases gestuelles ${ }^{6}$ (McNeill, 1992) distinctes, le geste produit de la main droite est indépendant de celui réalisé de la main gauche: les deux gestes ne partagent donc pas nécessairement la même fonction, ni la même dimension gestuelle.

Dans l'exemple ci-dessous ${ }^{7}$, la bimanualité est utilisée pour organiser différents foyers de prise de parole en FLS. La classe est en pleine discussion sur les élections présidentielles françaises. L'enseignante demande alors aux élèves le sens du mot "débat".

Corpus FLS/10 "Le débat, qu'est-ce que ça veut dire?

\begin{tabular}{|l|l|l|}
\hline 1 & E & le débat ++ qu'est-c(e) que ça veut dire $\uparrow$ \\
\hline 2 & Nolan & je sais pas \\
\hline 3 & E & tu sais pas $\uparrow$ \\
\hline 4 & Antonio & oui je sais \\
\hline 5 & E & tu sais vas-y on t'écoute \\
\hline 6 & Antonio & comme : : \\
\hline 7 & Nolan & deux personnes \\
\hline 8 & Antonio & les les personnes parl/e/ (au ?) \\
\hline 9 & E & des personnes parlent $\uparrow$ \\
\hline 10 & Nolan & des ++ des des \\
\hline 11 & E & tu brûles c'est bien c'est ça \\
\hline 12 & Nolan & des des \\
\hline 13 & Antonio & plusieurs choses euh : : une chose X \\
\hline 14 & E & mais plus précisément vas-y (à Nolan) $\uparrow$ \\
\hline 15 & Nolan & quand ++ deux ++ personnes elles parlent de une chose \\
\hline 16 & E & tout à fait ++ tout à fait deux personnes qui parlent du MÊME sujet \\
\hline
\end{tabular}

22 Au tour 4, Antonio se propose d'expliquer le terme après que Nolan a déclaré son incompétence en la matière (tour 2). Pourtant, à peine a-t-il entamé sa définition que Nolan intervient pour faire une proposition. L'enseignante valide de la main droite 
l'intervention d'Antonio (Fig.1, clichés a-d). Comme l'indique son geste déictique initié au cliché e, précédé du regard vers Nolan (cliché d), l'enseignante s'intéresse également rapidement à la demande de prise de parole qu'effectue cet élève verbalement (fauxdéparts aux tours 10 et 12) et gestuellement (la main levée, clichés a-g). Elle effectue donc un double geste main droite/main gauche : sa main droite oscille, on observe un léger mouvement du poignet (Calbris \& Montredon, 1986: 19); cet emblème symbolisant l'approximation vise à évaluer l'intervention d'Antonio. De sa main gauche, elle effectue un déictique pour attribuer la parole à Nolan. Fait intéressant, elle maintient son geste de la main droite, comme pour ne pas couper le lien avec Antonio. Cette mise en suspension du geste est une véritable ressource interactionnelle: tout en attribuant la parole à Nolan, l'enseignante conserve "visuellement" à l'esprit l'intervention d'Antonio, que celle de Nolan vient compléter. L'EnsM finit par rompre gestuellement le lien avec les élèves lorsqu'elle commence à symboliser par des gestes la parole de Nolan : "elles parlent" (tour 15, clichés $\mathrm{i}-\mathrm{j}$ ).

Figure 1 - Gestion bimanuelle des interventions en FLS

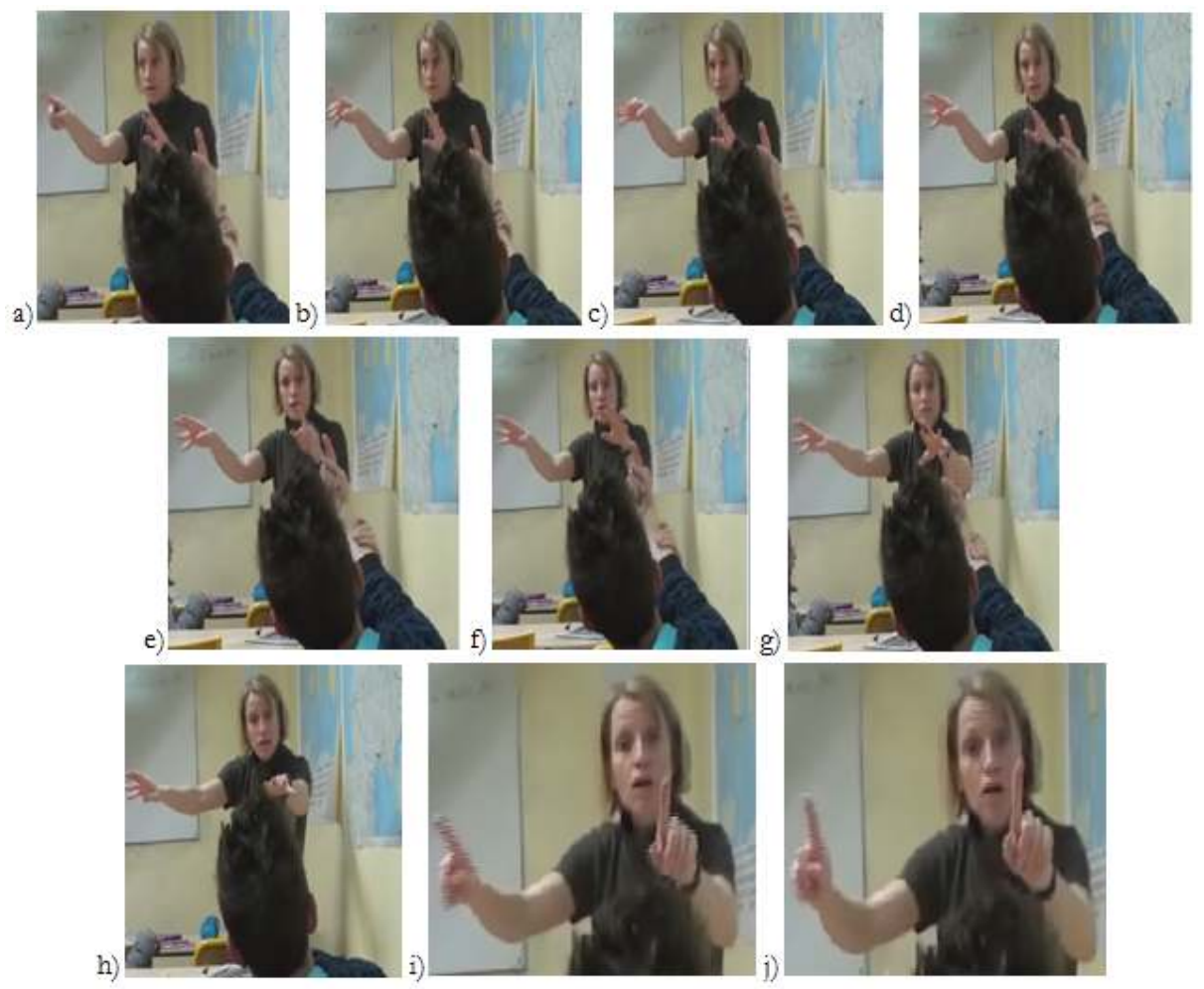

Nolan : [des des

Antonio : plusieurs choses euh : : une chose X

$\mathrm{E}:$ mais plus précisément vas-y (à Nolan)

Nolan : quand ++ deux ++ personnes elles parlent] de une chose

(tours 12-15, 19'54-19'58)

Ajoutons que dans ces situations où l'enseignante est impliquée dans plusieurs interactions simultanément, la bimanualité lui offre les moyens de construire une relation individuelle avec chacun de ses interlocuteurs principaux. Même si le groupe d'élèves allophones est plus réduit que celui de FL1, nous ne pouvons négliger l'impact que cette reconnaissance gestuelle peut avoir pour chacun d'entre eux. A fortiori dans une 
classe où l'effectif est plus important, cette stratégie peut avoir toute son utilité, notamment pour gérer les prises de parole intempestives tout en poursuivant une autre activité. C'est ce que nous montre l'exemple suivant ("S'il te plait, tu n'interviens pas") issu d'un corpus FL1. L'enseignante, professeure principale de cette classe, organise la prochaine réunion parents/professeurs. Un élève l'interpelle sur la question des bulletins. Alors qu'elle lui explique le fonctionnement, une autre élève, Loubna, intervient sans permission.

\section{Corpus FL1/1 "S'il te plait, tu n'interviens pas"}

\begin{tabular}{|l|l|l|}
\hline 1 & Youssef & euh c'est pas au second conseil de classe qu'on a le bulletin \\
\hline 2 & E & $\begin{array}{l}\text { non }++ \text { et au conseil de classe les professeurs donnent leur avis etc etc on voit [et } \\
\text { puis// }\end{array}$ \\
\hline 3 & Loubna & y'a les délégués aussi \\
\hline 4 & E & on donne \\
\hline 5 & Serge & y'a que les X \\
\hline 6 & E & $\begin{array}{l}\text { chut s'il te plait tu n'interviens pas (à Loubna) ++++ et le pro]fesseur principal c'est- } \\
\text { à-dire moi et bien je note ce l'avis euh : : : : l'avis général d'accord }\end{array}$ \\
\hline
\end{tabular}

La séquence de captures d'écran suivante (Fig.2) permet de visualiser l'évolution du rappel normatif, et d'en comprendre la motivation. De la main droite, nous voyons que l'enseignante entame un geste iconique sur "et puis" (tour 2) pour représenter l'acte d'écrire (Fig.2, clichés a-c). Or, nous nous apercevons que son affilié lexical (Schegloff, 1984 ; Kipp, Neff \& Albrecht, 2007), "je note" (tour 6), est situé bien plus loin après les interventions de Loubna et de Serge. Ainsi, même si la pause située après la première partie de l'intervention de l'enseignante (tour 2) a pu induire Loubna en erreur et lui faire croire qu'elle pouvait prendre la parole (tour 3), il s'avère que la professeure n'avait pas terminé son énoncé. C'est ce qu'indique le connecteur "et puis" et ce début de geste iconique suspendu pour accompagner le rappel normatif (clichés d-g). L'élève a donc interrompu l'enseignante dans son explication verbo-gestuelle. Celle-ci maintient son geste iconique (cliché $\mathrm{d}$ ) et entame un emblème de rejet (la paume est "levée contre l'extérieur", Calbris \& Montredon, 1986: 88) de la main gauche pour opposer un refus à Loubna (clichés d-j). Elle renforce la valeur illocutoire de ce geste par un rappel à l'ordre qu'elle appuie d'un regard fixe (cliché i). Elle récupère le fil de son explication après un "long" silence de 3 secondes en reprenant le geste iconique qu'elle avait dû abandonner plus tôt (clichés j-k). L'enseignante maintient toutefois l'emblème de rejet produit avec sa main gauche : 
Figure 2 - Gestion bimanuelle des interventions en FL1
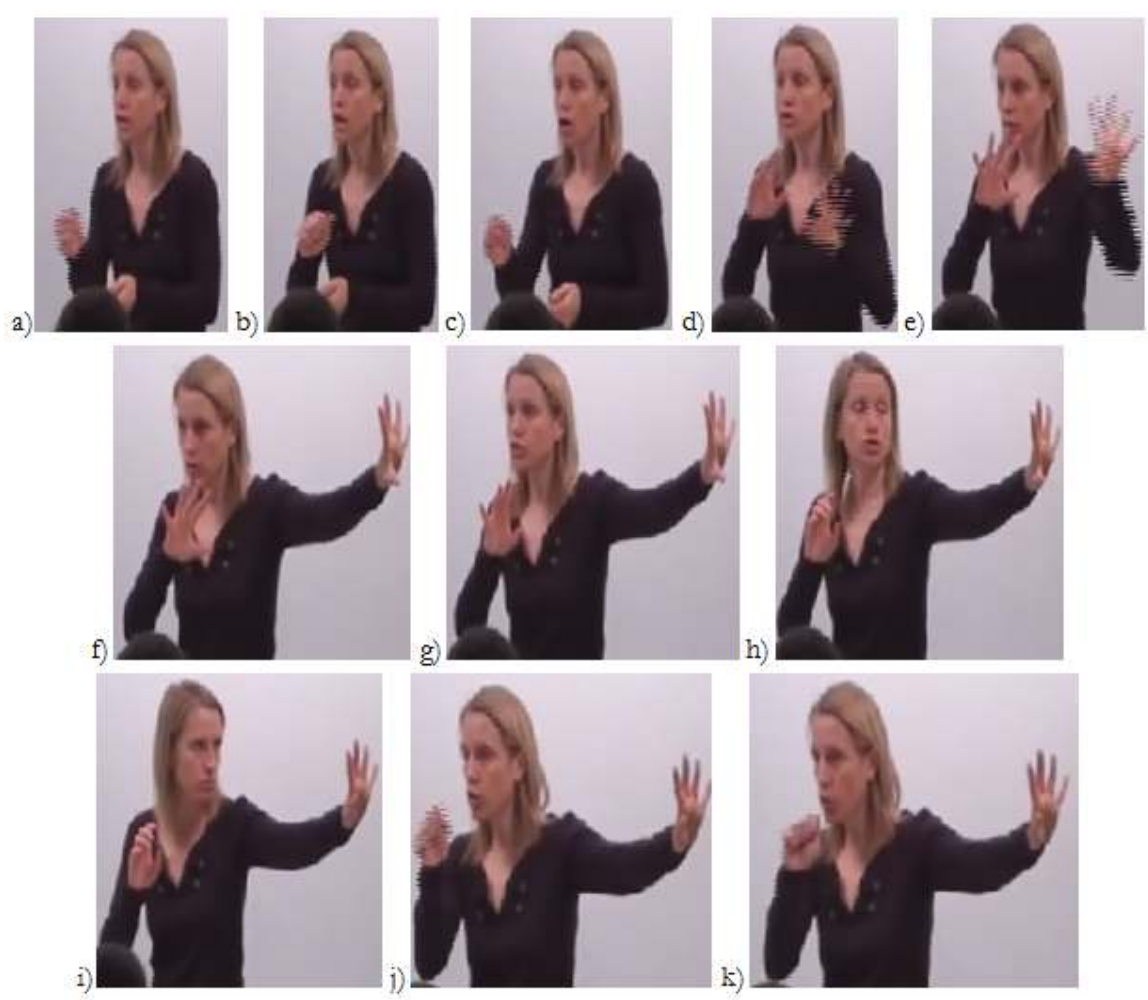

Tours 2-6 (7'03-7'10)

La suite de l'explication montre que l'enseignante poursuit ce geste encore quelques secondes alors même que Loubna, ni aucun autre élève d'ailleurs, ne tente de reprendre la parole.

\section{La bimanualité vue par l'enseignante : une double utilité pédagogique et psychologique}

Lors des séances d'autoscopie, cette enseignante a été sensible à ses gestes bimanuels, auxquels attribue une double intention pédagogique et psychologique

\section{Corpus autoscopie 3CA-FL1}

\begin{tabular}{|l|l|l|l|}
\hline 1 & E & $\begin{array}{l}\text { ma façon de gérer c'est je fais ça hop pour euh : taisez-vous ++ avec les gestes j'essaye } \\
\text { aussi de gérer aussi un petit peu + bon ici on se tait alors qui veut parler là de désigner } \\
\text { de- j'essaye avec les gestes c'est vrai que ça ça m'aide je sens bien de- mais même ça } \\
\text { m'aide personnellement quoi de de hop faire taisez-vous ici de désigner }\end{array}$ \\
\hline 2 & ENQ. & en quoi ça t'aide tellement de $::: \uparrow$ & \\
\hline
\end{tabular}




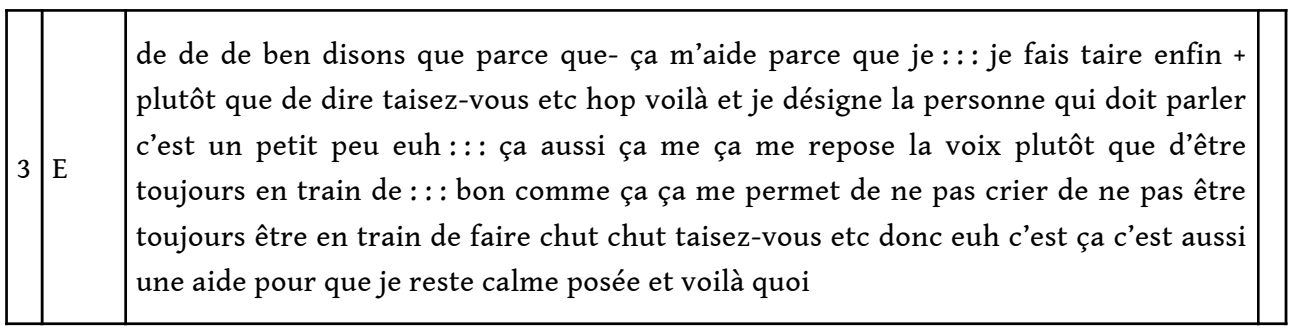

Sur le plan pédagogique, l'enseignante reconnait à la gestuelle une force illocutoire assimilable à la parole "je fais taire enfin plutôt que de dire taisez-vous" (tour 3), à la différence près qu'elle permet de gérer plusieurs actions à la fois. En effet, contrairement au verbal, "taisez-vous", qui induit spontanément un accompagnement du regard vers un élève imaginaire (Fig.3, cliché a) et une mono-activité puisque geste, parole et regard sont orientés dans la même direction, le geste bimanuel permet de dissocier deux actions et deux coénonciateurs : un coénonciateur principal, celui qu'elle désigne et regarde en même temps, et un coénonciateur "secondaire" que l'emblème gestuel tient littéralement à distance (clichés b-d).

Figure 3 - L'enseignante reproduisant son geste bimanuel durant une séance d'autoscopie

a)
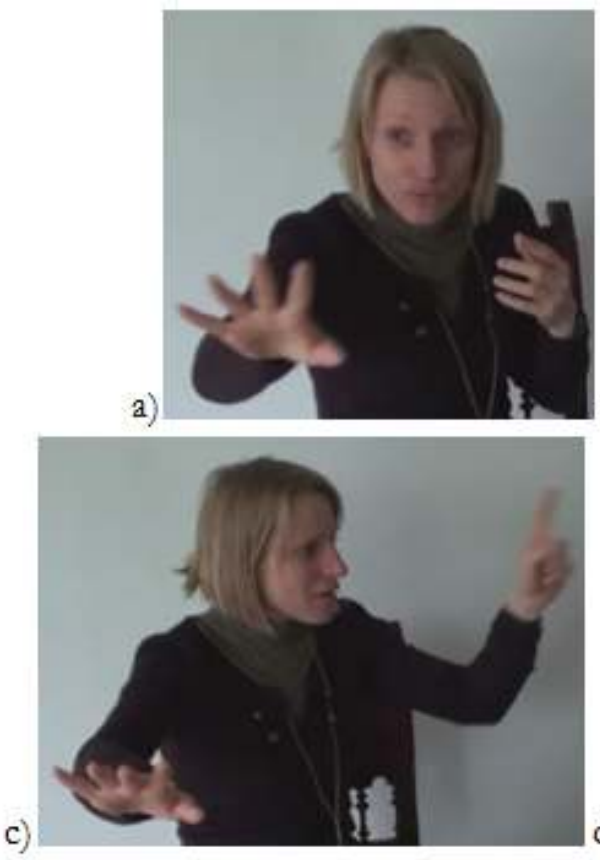

b)

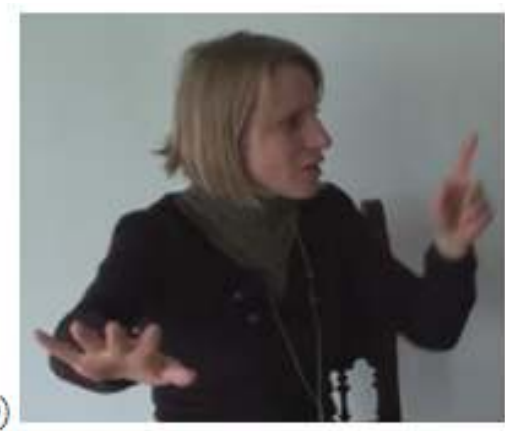

d)

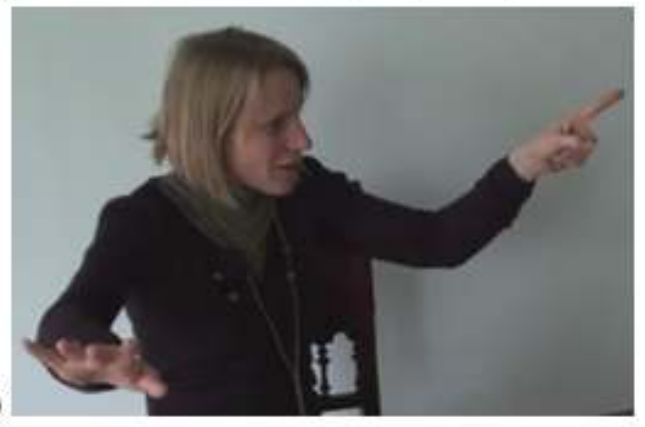

plutôt que de dire [taisez-vous etc. hop voilà] (tour 3)

exemples mentionnés par l'enseignante rappellent le caractère répétitif et usant de l'activité de gestion de classe. La bimanualité constitue un moyen semble-t-il plus simple ("hop", répété deux fois) et efficace ("voilà") pour organiser gestuellement les interactions de classe. L'intention psychologique est contenue dans un commentaire d'autant plus intéressant qu'inattendu. Ce procédé donne à l'enseignante le sentiment de moins se fatiguer. En se substituant à la parole, la bimanualité lui permet de reposer sa voix et de décroitre le rythme dans lequel elle est prise : "ça me permet de ne pas crier de ne pas être 
toujours en train de faire chut chut taisez-vous etc donc euh c'est ça c'est aussi une aide pour que je reste calme posée" (tour 3).

Ainsi, la bimanualité s'avère une stratégie essentielle dans la gestion des interventions multiples et parfois irrespectueuses des règles de prise de parole en classe. Elle permet également à l'enseignante d'organiser plusieurs activités à la fois, tout en se ménageant. Lorsque l'on s'intéresse de plus près à ces stratégies mises en œuvre lors des divers chevauchements qui ponctuent les interactions, nous nous apercevons que l'enseignante se démultiplie pour pouvoir considérer les interventions de plusieurs élèves à la fois. C'est sur cette ubiquité coénonciative, une stratégie foncièrement multimodale, que nous allons à présent nous pencher.

\section{Ubiquité coénonciative et tropes communicationnels}

31 Sur le plan interactionnel, nous avons récemment montré (Azaoui, 2014d) tout l'intérêt d'une approche multimodale de l'agir professoral (Cicurel, 2011). Notre analyse intégrée de gestes de pointage et du regard des enseignantes a mis au jour une compétence mimogestuelle que nous avons nommée "ubiquité coénonciative" (Azaoui, 2014d : 123). Elle renvoie à la capacité à être le coénonciateur de plusieurs élèves quasi simultanément. L'enseignante peut ainsi faire respecter les normes interactionnelles tout en poursuivant simultanément une autre action, ou sélectionner parmi les diverses interventions concomitantes celle qui correspond à ses attentes linguistiques/pédagogiques. Plus globalement, les interactions didactiques étant foncièrement polylogales, l'enseignante peut être amenée à valider l'intervention d'un élève 1 par un hochement de tête, tout en désignant de la main un élève 2 pour lui donner la parole et répondre, dans la foulée, à un élève 3. Cette aptitude, qui pourrait fort bien se retrouver dans d'autres contextes professionnels, met nécessairement en œuvre des stratégies multimodales de gestion des interactions. À l'origine de cette notion se trouve notre intérêt pour une action disjointe regard/geste observée chez les enseignantes de nos corpus. Nous l'avons notée essentiellement dans le corpus de l'EnsT, même si à l'occasion elle est mise en œuvre par l'EnsM, comme dans ce premier exemple ("Ah très bien Nolan").

Dans ce cours de FLS, il est question des élections présidentielles en France et de la campagne des candidats. Nolan, un élève assez faible en français, intervient et soumet une proposition que valide l'enseignante. Dans cet extrait, les élèves donnent l'impression d'être en concurrence pour proposer la bonne réponse alors que l'enseignante ne semble pas être dans l'attente d'une réponse précise. Chacun avance une idée, dont l'enseignante (tours 9 et 11) ou les élèves (tour 10) évaluent la pertinence. L'intervention de Nolan au tour 12 est validée avec emphase. La particularité de cette validation n'est toutefois pas cette amplification prosodique ; elle se situe au niveau mimogestuel.

\section{Corpus M-FLS/10 "Ah très bien Nolan" (16’01-16’28)}

\begin{tabular}{|c|c|c|}
\hline 1 & E & $\begin{array}{l}\text { euh : en c(e) moment qu'est-c(e) qui s(e) passe avant le premier tour } \uparrow \text { qu'est-c(e) } \\
\text { qui s(e) passe } \uparrow++ \text { que } s(e) \text { passe-t-il } \uparrow\end{array}$ \\
\hline 2 & Anouar & il a :::::: \\
\hline 3 & E & que font les hommes politiques avant ++ avant les élections $\uparrow$ \\
\hline
\end{tabular}




\begin{tabular}{|l|l|l|}
\hline 4 & Anouar & $<$ XX> \\
\hline 5 & E & qu'est-c(e) qu'ils font les hommes politiques $\uparrow$ \\
\hline 6 & Anouar & euh $:::$ \\
\hline 7 & E & oui $\uparrow$ \\
\hline 8 & Carlos & ils réfléchissent \\
\hline 9 & E & ils réfléchissent ++ tout à fait \\
\hline 10 & Anouar & non c'est pas ils réfléchissent \\
\hline 11 & E & ils réfléchissent à un programme \\
\hline 12 & Nolan & il y a publicité \\
\hline 13 & E & AH :: : : : : TRÈs bien Nolan \\
\hline 14 & Antonio & il a dit quoi $\uparrow$ \\
\hline 15 & E & tu peux répéter $\uparrow$ \\
\hline 16 & Nolan & publicité \\
\hline 17 & Anouar & quoi $\uparrow$ \\
\hline 18 & E & publicité c'est ça qu(e) t'as dit $\uparrow++$ voilà \\
\hline
\end{tabular}

Les clichés suivants permettent de visualiser cette polyactivité qui s'étend sur trois secondes. Au moment où l'enseignante formule ses félicitations "ah très bien", elle pointe son index vers Nolan et le regarde (Fig.4, clichés a-d). Fait singulier, à l'instant où elle prononce son prénom (15'21), elle oriente son regard vers les élèves situés à sa droite tout en maintenant son déictique en direction de Nolan (cliché e).

Figure 4 - Exemple de disjonction regard/déictique

a)

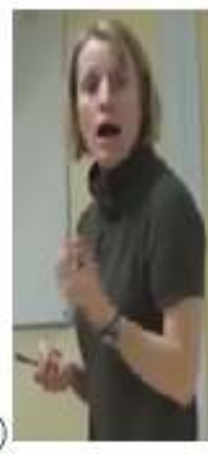

b)

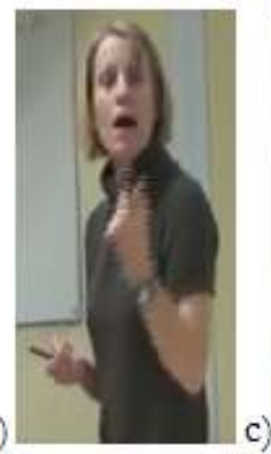

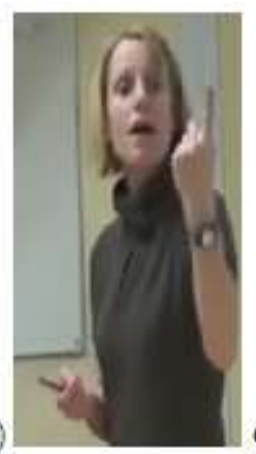

d)

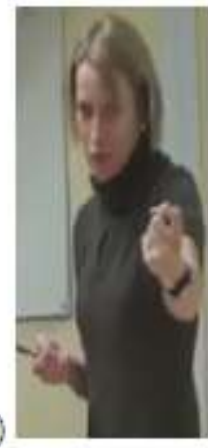

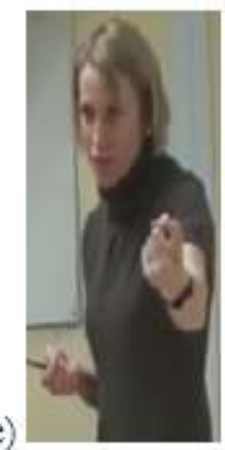

[Ah très bien Nolan] (tour 13, 15'19-15'22) 


\section{Brun :}

\section{Corpus T-FL1/1-2 "C'est monsieur tout le monde" (6'20-6'31)}

\begin{tabular}{|l|l|l|}
\hline 1 & E & alors pourquoi la cinquantaine toi (à Pierre $) \uparrow$ \\
\hline 2 & Albert & [(c'est monsieur tout le monde ?) \\
\hline 3 & Pierre & non mais j(e) sais pas la cinquantaine la soixantaine j'en sais rien \\
\hline 4 & E & XX ++ oui Albert] un peu plus fort \\
\hline 5 & Albert & c'est monsieur tout le monde \\
\hline 6 & E & oui ++ c'est monsieur tout le monde \\
\hline
\end{tabular}

La séquence d'actions mimogestuelles décrites ici se réalise en l'espace de cinq secondes (cf. durée des clichés reproduits, Fig.5). Tout d'abord, l'enseignante se positionne comme l'interlocutrice de Pierre qu'elle vient d'interpeler (tour 1) et vers lequel elle oriente son regard (Fig.5, cliché a). Pourtant très rapidement, elle porte de l'intérêt à l'intrusion d'Albert (clichés b-f) : tout en écoutant et en regardant Pierre, elle pointe Albert du doigt pour valider son intrusion ${ }^{8}$. 
Figure 5 - Exemple d'ubiquité coénonciative en FL1
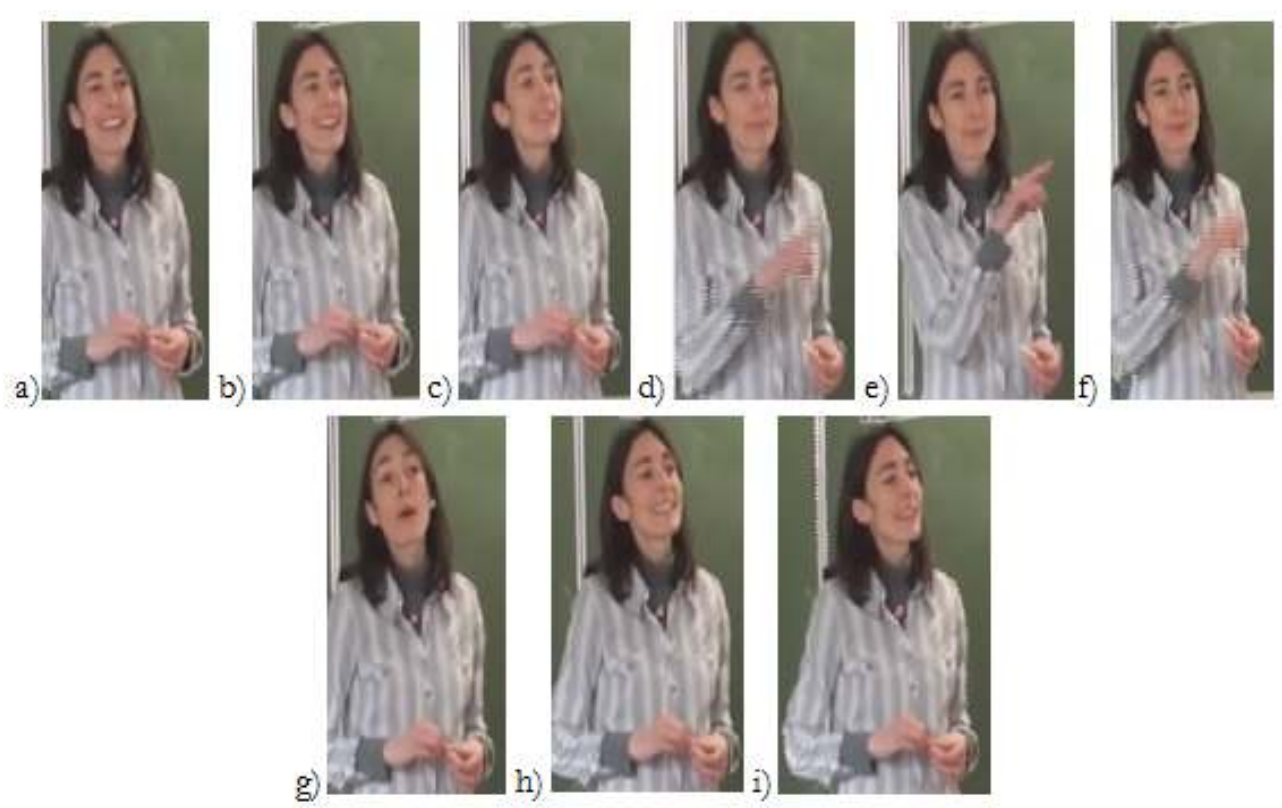

Albert : (c'est monsieur tout le monde?)

Pierre : non mais $\mathrm{j}(\mathrm{e})$ sais pas la cinquantaine la soixantaine $\mathrm{j}$ 'en sais rien $\mathrm{E}: \mathrm{XX}++$ oui Albert

(tours 2-4, 6'22-6'27)

Cela interroge à nouveau sur l'identité de son coénonciateur "privilégié" (KerbratOrecchioni, 1990 : 88). Si les analyses de Goodwin $(1981,1996)$ ou Goffman (cité dans Kerbrat-Orecchioni, ibid.) ont pu mettre au jour le rôle du regard dans la construction du coénonciateur, il se trouve que cela ne peut s'appliquer dans cette situation. L'hypothèse d'avoir deux coénonciateurs simultanés chacun signalé par un canal de communication (regard et déictique) peut évidemment être envisagée. Se pose quoi qu'il en soit la question de la hiérarchie des destinataires. Le déictique, sur lequel l'enseignante effectue un battement pour signaler l'intérêt qu'elle porte à l'énoncé d'Albert qu'elle désigne du doigt (clichés d-e), nous indique, à notre sens, que son coénonciateur principal est Albert ; cela implique l'existence d'un trope communicationnel. Cette interprétation est étayée par la suite de l'échange. En effet, c'est à peine si l'intervention de Pierre est reconnue : dès qu'il a prononcé "non mais $j(e)$ sais pas" (tour 3), l'enseignante valide gestuellement l'énoncé qu'Albert vient de terminer (clichés d-f). La remarque de Pierre ne semble pas suffisamment pertinente pour qu'elle s'y attarde : elle émet un commentaire, inaudible, et revient aussitôt vers Albert.

\section{Conclusion}

Le propos de cette contribution était de souligner le rôle de la multimodalité dans l'action professorale. En nous appuyant sur un double corpus de films de classe et de vidéoscopies, nous avons montré comment les enseignantes faisaient appel à des ressources multimodales dans le feu de leurs actions pour gérer les interventions multiples. 
Appréhendée dans une perspective étique, la bimanualité permet d'organiser les prises de parole simultanées. L'entretien vidéoscopique mené avec l'enseignante concernée permet de confirmer cette première analyse d'un point de vue émique. Il offre l'occasion d'apprécier cette ressource autrement, en considérant une dimension plus égocentrée, orientée vers la gestion de la fatigue vocale et physique de l'enseignante.

Nos résultats ont également permis de revenir sur la notion d'ubiquité coénonciative à travers le prisme des implications énonciatives possibles de cette ressource. Nous nous sommes tout particulièrement intéressé ici à la question du trope communicationnel que l'ubiquité coénonciative pouvait induire. Ce renversement de hiérarchie du destinataire, produit de l'interaction geste/regard, donne à l'enseignante l'opportunité de signaler à ses élèves l'intervention la plus pertinente selon ses attentes; cette pertinence étant évaluée à l'aune du projet pédagogique.

Ces résultats montrent l'intérêt, voire la nécessité, de considérer de manière encore plus approfondie la multimodalité de l'agir professoral en classe, où l'enseignant est considéré dans cet espace fondamentalement plurisémiotique et plurivocal. La connaissance du geste pédagogique, notamment, s'enrichira de ces paramètres.

\section{BIBLIOGRAPHIE}

Adams, T. W. (1998). Gestures in foreigner talk. (Thèse de doctorat non publiée). Pennsylvania: University of Pennsylvania.

Allen, L. Q. (1995). "The effects of emblematic gestures on the development and access of mental representations of French expressions". The Modern Language Journal, vol. 79. pp. 521-529.

Azaoui, B. (2014a). Coconstruction de normes scolaires et contextes d'enseignement. Une étude multimodale de l'agir professoral. (Thèse de doctorat non publiée). Montpellier : Université Paul Valery, Montpellier 3.

Azaoui, B. (2014b). "Quels apports de la vidéo en formation des enseignants de français ?". Diversité, $\mathrm{n}^{\circ}$ 177. pp. 130-134.

Azaoui, B. (2014c). "Très bien, super, génial et $\mathrm{C}^{\mathrm{ie}}$. Impact du contexte classe sur les félicitations multimodales de l'enseignant". In Aguilar Rio, J., Brudermann, C. \& Leclère, M. (dir.). Langages, cultures, sociétés : interrogations didactiques. Paris : Riveneuve. pp. 47-74.

Azaoui, B. (2014d). "Multimodalité des signes et enjeux énonciatifs en classe de FL1/FLS". In Tellier, M. \& Cadet, L. (dir.). Le corps et la voix de l'enseignant : mise en contexte théorique et pratique. Paris : Éditions Maison des Langues. pp. 115-126.

Barrier, G. (2004). "Gestes performatifs, expression faciale et partage attentionnel : analyse de la poursuite oculaire à partir d'une scène conversationnelle". Semiotica, nº 152. pp. 217-233.

Bigot, V., Blondel, E., Cadet, L. \& Causa, M. (2004). "La construction du répertoire d'enseignement lors du passage du statut d'étudiant à celui d'enseignant de FLE". Marges linguistiques, ${ }^{\circ} 7$. Version auteur, sans pagination. 
Bouchard, R. (1998). "L’interaction en classe comme polylogue praxéologique". Mélanges en hommage à Michel Dabène. Grenoble : Ellug. Accessible en ligne. http://www.inrp.fr/colloques/ seminaires/2004/sem_didac/bouchard_article_1.pdf.

Cadet, L. (2006). "Des notions opératoires en didactique des langues et des cultures : Modèles ? Représentations? Culture éducative ? Clarification terminologique". Les Cahiers de l'Acedle, $\mathrm{n}^{\circ} 2$. pp. 36-51. Disponible en ligne. http://acedle.org/IMG/pdf/Cadet-L_cah2.pdf.

Cadet, L \& Causa, M. (2005). "Culture(s) éducative(s) et construction d'un répertoire didactique en formation initiale". In Beacco, J.-C. Chiss, J.-L. Cicurel, F. \& Véronique, D. (dir.). Les cultures éducatives et linguistiques dans l'enseignement des langues. Paris : Presses Universitaires de France. pp. 158-181.

Calbris, G. \& Montredon, J. (1986). Des gestes et des mots pour le dire. Paris : Clé international.

Calbris, G. \& Porcher, L. (1989). Geste et communication. Paris : Didier.

Causa, M. (2012). "Le répertoire didactique : une notion complexe". In Causa, M. (dir.). Formation initiale et profils d'enseignants de langues : enjeux et questionnements. Bruxelles : De Boeck. pp. 15-72.

Cicurel, F. (2002). "La classe de langue, un lieu ordinaire, une interaction complexe". Acquisition et interaction en langue étrangère (Aile), $\mathrm{n}^{\circ}$ 16. pp. 145-164. Disponible en ligne. http:// aile.revues.org/801.

Cicurel, F. (2005). "La flexibilité communicative : un atout pour la construction de l'agir enseignant". Le Français dans le monde. Recherches \& applications, numéro spécial juillet. pp. 180-191.

Cicurel, F. (2011). Les interactions dans l'enseignement des langues. Agir professoral et pratiques de classe Paris : Didier.

Clanet, J. (2002). "Gestion et organisation de l'interaction maitre-élèves". In Bressoux, P. et al. (dir.). Les stratégies de l'enseignant en situation d'interaction. Note synthèse pour Cognitique Programme Ecole et Sciences Cognitives. Paris : Ministère de la jeunesse de l'éducation et de la recherche. pp. 77-108. Disponible en ligne http://edutice.archives-ouvertes.fr/docs/00/00/17/90/PDF/ Bressoux.pdf.

Condon, W. (1976). "Une analyse de l'organisation comportementale". In Cosnier, J. \& Brossard, A. (dir.) (1984). La communication non verbale. Paris : Delachaux et Niestlé.

Cook, J. (1977). "Regard et regard réciproque dans les interactions verbales". In Cosnier, J. \& Brossard, A. (dir.) (1984). La communication non verbale. Paris : Delachaux et Niestlé. pp. 125-144.

Corraze, J. (1980). Les communications non-verbales. Paris : Presses Universitaires de France.

Dabène, L. (1984). Pour une taxinomie des opérations métacommunicatives en classe de langue étrangère. Etudes de Linguistiques Appliquées (ELA), 55. pp. 39-46.

Ekman, P. \& Friesen, W. V. (1969). "Nonverbal leakage and clues to deception". Psychiatry journal for the study of interpersonal processes, vol. 32, $\mathrm{n}^{\circ}$ 1. pp. 88-106.

Foerster, C. (1990). "Et le non-verbal ?" In Dabène, L., Cicurel, F., Lauga-Hamid, M.-C. \& Foerster, C. (dir.). Variations et rituels en classe de langue. Paris : Hatier. pp. 72-93.

Goodwin, C. (1981). Conversational organization. Interaction between speakers and hearers. London: Academic press.

Goodwin, C. (1996). "Transparent vision". In Ochs, E., Schegloff, E. A. \& Thompson, S. A. (dir.). Interaction and grammar. Cambridge: Cambridge University Press. pp. 370-404. 
Grosjean, M. \& Traverso, V. (1998). "Les cadres participatifs dans les polylogues : problèmes méthodologiques". In Cabasino, F. (dir.). Du dialogue au polylogue. Rome : Cisu. pp. 51-67.

Heylen, D. (2005). "A closer look at gaze". In Pelachaud, C., André, E., Kopp, S. \& Ruttkay Z. (dir.). Creating bonds with embodied conversational agents: Aamas workshop. Utrecht : University of Utrecht. pp. 3-9. Disponible en ligne http://eprints.eemcs.utwente.nl/1869/01/gaze-aamas.pdf.

Heylen, D., Bevacqua, E., Tellier, M. \& Pelachaud, C. (2007). "Searching for prototypical facial feedback signals". In Aylett, R., Krenn, B., Pelachaud, C. \& Shimodaira, H. (dir.). Intelligent Virtual Agents. Lecture Notes in Computer Science. Berlin: Springer Verlag. pp. 147-153.

Joly, I. (2011). Le corps sans représentation. De Jean-Paul Sartre à Shaun Gallagher. Paris: L'Harmattan.

Kååntå, L. (2007). "Pointing, underlining and gaze as resources of instructional action in classroom interaction". In Mondada, L. \& Markaki, V. (dir.). Interacting bodies. Online proceedings of the $2 d$ ISGS Conference. Disponible en ligne (version numérique non paginée). http://gesturelyon2005.ens-lyon.fr/IMG/pdf/Kaanta.pdf.

Kellerman, S. (1992). "'I see what you mean': the role of kinesic behaviour in listening and implications for foreign and second language learning". Applied linguistics, vol. 13, n 3. pp. 239-258.

Kendon, A. (1967). "Some functions of gaze-direction in social interaction". Acta Psychologica, vol. 26. pp. 22-63.

Kerbrat-Orecchioni, C. (1990). Les interactions verbales, Tome I. Paris : Armand Colin.

Kerbrat-Orecchioni, C. (1999). L'énonciation. De la subjectivité dans le langage. Paris : Armand Colin. Kerbrat-Orecchioni, C. \& Plantin, C. (1995). Le trilogue. Lyon : Presses Universitaire de Lyon.

Kipp, M.; Neff, M. \& Albrecht, I. (2007). "An annotation scheme for conversational gestures: how to economically capture timing and form". Journal on language resources and evaluation, vol. $41, \mathrm{n}$ -3-4. pp. 325-339.

Lazaraton, A. (2004). "Gestures and speech in the vocabulary explanations of one ESL teacher: a microanalytic inquiry". Language learning, vol. 54, $\mathrm{n}^{\circ}$ 1. pp. 79-117.

McNeill, D. (1992). Hands and mind: what gestures reveal about thought. Chicago : University of Chicago Press.

Moirand, S. (1993). "Autour de la notion de didacticité". Les Carnets du Cediscor, n 1. pp. 9-20. Disponible en ligne http://cediscor.revues.org/600.

Rivière, V. \& Bouchard, R. (2011). "Une compétence professionnelle de l'enseignant : gérer la simultanéité des interactions". Colloque international Inrp-ENS, Lyon. Disponible en ligne. http:// www.inrp.fr/archives/colloques/travail-enseignant/contrib/112.htm.

Schachter, J. (1981). "The hand signal system". Tesol Quarterly, vol. 15, n 2. pp. 125-138.

Schegloff, E. A. (1984). "On some gestures' relation to talk". In Heritage, J. (dir.). Structures of social action. Cambridge: Cambridge University Press. pp. 266-298.

Sime, D. (2008). "'Because of her gesture, it's easy to understand'. Learners' perception of teachers' gestures in the foreign language class". In McCafferty, S. G. \& Stam, G. (dir.). Gesture: second language acquisition and classroom research. New-York, NY: Routledge. pp. 259-279.

Sloetjes, H. \& Wittenburg, P. (2008). "Annotation by category - Elan and ISO DCR". In Calzolari, N. et al. (dir.). Proceedings of the 6th International conference on language resources and evaluation. 
Marrakech : European Language Resources Association. pp. 816-820. Disponible en ligne. http:// lrec-conf.org/proceedings/lrec2008/pdf/208_paper.pdf.

Streeck, J. (1993). "Gesture as communication I: its coordination with gaze and speech". Communication monographs, vol. 60, $\mathrm{n}^{\circ} 4$. pp. 275-299.

Taleghani-Nikzam, C. (2008). "Gestures in foreign language classrooms: an empirical analysis of their organization and function". In Bowles, M., Foote, R., Perpiñan, S. \& Bhatt, R. (dir.). Selected proceedings of the 2007 second language research forum. Somerville, MA: Cascadilla. pp. 229-238. Disponible en ligne. http://www.lingref.com/cpp/slrf/2007/paper1747.pdf.

Tellier, M. (2006). L'impact du geste pédagogique sur l'enseignement-apprentissage des langues étrangères : Etude sur des enfants de 5 ans. (Thèse de doctorat non publiée). Paris : Université Paris 7-Denis Diderot. Disponible en ligne. https://halshs.archives-ouvertes.fr/tel-00371041

Tellier, M. (2008a). "The effect of gestures on second language memorisation by young children". Gesture, vol. 8, n² 2.pp. 219-235.

Tellier, M. (2008b). "Dire avec des gestes". Le Français dans le monde. Recherches \& applications, $\mathrm{n}$ 44. pp. 40-50.

Tellier, M. (2009). "Usage pédagogique et perception de la multimodalité pour l'accès au sens en langue étrangère". In Bergeron, R., Plessis-Bélair, G. \& Lafontaine, L. (dir.). La place des savoirs oraux dans le contexte scolaire d'aujourd'hui. Montréal : Presses Universitaires du Québec. pp. 223-245.

Tellier, M. (2010). "Faire un geste pour l'apprentissage : le geste pédagogique dans l'enseignement précoce". In Corblin, C. \& Sauvage, J. (dir.). L'enseignement des langues vivantes étrangères à l'école. Impact sur le développement de la langue maternelle. Paris : L'Harmattan. pp. 31-54.

Tellier, M., Azaoui, B. \& Saubesty, J. (2012). "Segmentation et annotation du geste : méthodologie pour travailler en équipe". In Braffort, A., Boutora, L. \& Sérasset, G. (dir.). Actes de la conférence conjointe JEP-Taln-Recital 2012 : Atelier Défi GEste Langue des Signes (Degels), 4-8 juin 2012, Grenoble, France. Grenoble : Afcp/Atala. pp. 41-55.

Vion, R. (1992). La communication verbale. Analyse des interactions. Paris : Hachette.

Watzlawick, P., Helmick Beavin, J. \& Jackson, D. (1972). Une logique de la communication. Paris : Seuil.

Winkin, Y. (1981). La nouvelle communication. Paris : Seuil.

\section{ANNEXES}

\section{Conventions de transcription}

$::=$ allongement syllabique plus ou moins long

$++=$ pause plus ou moins longue

(e) = son non prononcé

$\mathrm{XX}=$ segment de parole inaudible

(mais ?) = transcription incertaine

m- = auto-interruption 
MAjuscule = emphase vocalique sur la partie écrite en majuscule

\section{$/ /=$ interruption}

segment souligné (simple, double ou autre variante) = chevauchement de paroles

(sourires) $=$ informations complémentaires venant éclairer le propos

$\uparrow=$ voix ascendante

$\downarrow=$ voix descendante

[...] = segment de l'énoncé sur lequel est produit le geste

\section{NOTES}

1. Version numérique non paginée.

2. Enseignement d'une discipline non linguistique (mathématiques, histoire-géographie, éducation physique et sportive, etc.) en langue étrangère.

3. C'est Moirand qui met en gras ces termes.

4. Joly emploie le terme dans le cadre d'une réflexion philosophique du corps amputé.

5. Logiciel libre de droits, développé par le Max Planck Institute de Nimègue, Pays-Bas.

6. Une unité gestuelle est constituée de plusieurs mouvements gestuels produits avec la parole.

7. Tous les prénoms ont été modifiés.

8. Notons également que l'enseignante réalise ici une forme d'infraction interactionnelle en se désintéressant ponctuellement de l'intervention de Pierre.

\section{RÉSUMÉS}

Il est aujourd'hui admis que les évènements de la classe s'inscrivent dans des interactions polylogales et polyfocalisées. Il revient à l'enseignant de gérer la simultanéité de ces échanges en ayant recours à toute une série de ressources multimodales. Dans la présente contribution, nous nous intéressons essentiellement aux actions professorales mimogestuelles. Nos analyses mettent au jour deux ressources, la bimanualité et l'ubiquité coénonciative qui servent les intentions pédagogiques des enseignantes de notre corpus. Si nous nous situons à un autre niveau d'analyse, ces ressources nous invitent également à nous interroger sur les enjeux énonciatifs qu'elles induisent.

Classroom interactions are intrinsically polyphonic in the sense that they not only gather more than three participants but they also are polyfocal. Consequently, the teacher is supposed to manage these simultaneous interventions to carry out his pedagogical planning. It appears that his verbal resources do not suffice to deal with this polyphonic reality of the class. This paper aims at analyzing the multimodal resources teachers resort to. Our results show that the teachers from our corpora use two sorts of resources we named bimanuality and co-enunciative ubiquity. These findings also raise questions related to the enunciative implications these mimo-gestural actions trigger. 
INDEX

Mots-clés : polyfocalisation, multimodalité, geste pédagogique, bimanualité, ubiquité coénonciative, trope communicationnel

Keywords : polyfocalisation, multimodality, teaching gesture, bimanuality, co-enunciative ubiquity, communicational trope

\section{AUTEUR}

\section{BRAHIM AZAOUI}

Université Paul Valéry - Montpellier III, Praxiling Brahim Azaoui est docteur en sciences du langage, enseignant titulaire d'anglais et de FLS et directeur de la plateforme d'accueil des élèves allophones nouvellement arrivés sur Montpellier (Espace L.S. Senghor).

Toile : http://www.praxiling.fr/azaoui-brahim.html.

Courriel : brahim.azaoui[at]gmail.com 